\title{
ANÁLISE TRIDIMENSIONAL DE ELEMENTOS FINITOS DA DISTRIBUIÇÃO DE TENSÕES EM IMPLANTES INCLINADOS: UMA REVISÃO DE LITERATURA
}

\section{THREE-DIMENSIONAL FINITE ELEMENT ANALYSIS OF STRESS DISTRIBUTION IN TILTED IMPLANTS: A REVIEW OF LITERATURE}

\author{
Gustavo Javier Salazar Littuma* \\ Camilo Andres Villabona Lopez** \\ Madalena Lucia Pinheiro Dias Engler*** \\ Ricardo de Souza Magini**** \\ Eduardo Meurer"****
}

\section{RESUMO}

Implantes inclinados atualmente são utilizados como uma alternativa cirúrgica e protética. Por tanto, estudos in vitro demonstram que o seu uso aumenta a concentração de tensão no osso cortical peri-implantar e questionase se esse fator pode levar ao insucesso da reabilitação oral estética. O objetivo do presente artigo foi avaliar a informação relacionada com a influência de forças funcionais sobre a biomecânica de implantes inclinados nas reabilitações orais implantossuportadas. No intuito de investigar essa hipótese, foi realizada uma revisão da literatura e busca de informações existentes em artigos científicos indexados ao PubMed, que avaliassem o efeito da distribuição das tensões na interface osso-implante de implantes inclinados, através do método de análise dos elementos finitos. Os resultados mostraram que o uso de implantes inclinados aumenta a concentração de tensões no osso cortical peri-implantar sob simulação de cargas parafuncionais. No entanto, análises in-vitro não podem prever de forma precisa o comportamento dos tecidos biológicos. Apesar dessa limitação dos estudos, pode-se concluir que elevados picos de tensões poderiam comprometer os limites de resistência do osso cortical, podendo levar a falhas do conjunto osso-implante. Todavia, quando associados a reabilitações de próteses múltiplas, os implantes inclinados mudam sua biomecânica, favorecendo a distribuição e redução das tensões e o aumento da estabilidade das reabilitações como um todo.

Descritores: Implantes dentários • Próteses e implantes • Reabilitação bucal • Análise de elementos finitos.

\section{ABSTRACT}

Tilted implants are currently used as a surgical and prosthetic's alternative. Therefore, in-vitro studies demonstrate that their use increases the stress concentration in the peri-implant cortical bone and it wonders whether this can lead to the failure of an aesthetic oral rehabilitation. The aim of this paper was to assess information related to the influence of functional forces on the biomechanics of tilted implants in implant oral rehabilitation. In order to investigate this hypothesis, a literature review was made in scientific articles indexed to PubMed, to assess the effect of the stress distribution in the bone-implant interface of tilted implants, through the analysis of the finite element method. The results showed that the use of tilted implants increases the concentration of stresses in the peri-implant cortical bone under the simulation of parafunctional loads. However, in-vitro tests may not accurately predict the behavior of biological tissues. Despite this limitation of the study, it can be concluded that high peaks of tension could compromise the strength limits of cortical bone, leading to failure of the bone-implant joint. However, when associated with multiple rehabilitations, the titled implants change their biomechanics, favoring the distribution and reduction of tension and the increase of the stability of restorations as a whole.

Descriptors: Dental implants • Prostheses and implants • Mouth rehabilitation • Finite element analysis.

* Mestrando em Implantodontia - Universidade Federal de Santa Catarina, Florianópolis, Brasil - E-mail: xaviersl_24@hotmail.com

** Professor da Universidade de Santo Tomás, Bucaramanga, Colômbia; Doutorando em Implantodontia - Universidade Federal de Santa Catarina, Florianópolis, Brasil; Estudante de pós-graduação em nível de especialização em Implantodontia - Avantis/UNIQUE, São José, Brasil e Periodontia da Faculdade São Leopoldo Mandic, Curitiba, PR, Brasil.

*** Mestranda em Implantodontia - Universidade Federal de Santa Catarina, Florianópolis, Brasil.

**** Professor titular - Universidade Federal de Santa Catarina, Florianópolis, Brasil.

***** Professor do Instituto de Pós-Graduação e Atualização em Odontologia - IPENO. 


\section{N T RO DUÇÃ O}

O uso de implantes osseointegrados para tratar o edentulismo total e parcial tem se convertido numa modalidade terapêutica perfeitamente integrada na odontologia restauradora ${ }^{1}$.

Na dentição natural, o ligamento periodontal tem a capacidade de absorver forças e permitir movimentações dos dentes, estabilizando a estrutura óssea adjacente. Entretanto, na interface osso-implante, não há possibilidade de movimentos ou de absorção das forças provenientes de cargas parafuncionais, fazendo com que o osso adjacente ao implante seja um local que recebe as forças diretamente e isso se torna maléfico à manutenção da crista óssea ${ }^{2}$.

Um fator chave para o sucesso ou insucesso na utilização de implantes é a forma como as forças são transferidas para o osso peri-implantar ${ }^{3}$. Porém, existem inúmeros fatores que afetam a distribuição de forças na interface osso-implante, sendo eles: o tipo de carga recebida (funcionais ou parafuncionais); as propriedades dos materiais com que foram confeccionados o implante e a prótese; a geometria e estrutura da superfície do implante; a qualidade e quantidade de osso circundante e a natureza da interface osso-implante ${ }^{4}$.

A carga oclusal sempre será transferida para os implantes e, em consequência disso, para o osso adjacente. Por isso, o desenho da prótese implantossuportada (biomecânica) também tem influência na manutenção óssea em torno dos implantes dentais ${ }^{5}$.

A análise dos elementos finitos (AEF) tornou a representação das forças possível e precisa, sendo considerada um método útil para prever o desempenho biomecânico dos implantes dentais, determinar o comportamento e efetividade de uma estrutura, e representar a maneira como um componente é submetido a um determinado carregamento ${ }^{3}$.

Reabsorção óssea, pneumatização dos seios maxilares, superficialização do forame mentual, canal mandibular e de estruturas anatômicas associadas, além de quantidade e qualidades ósseas deficientes, são fatores que podem limitar ou impedir a instalação de implantes. Nesse contexto, o uso de implantes inclinados é uma alternativa viável ${ }^{6}$.

Considerando-se que a utilização de implantes inclinados é uma alternativa cirúrgica e protética vastamente utilizada na atualidade, o objetivo deste artigo foi avaliar evidências publicadas relacionadas à influência de forças parafuncionais sobre a biomecânica de implantes inclinados em reabilitações implantossuportadas, no intuito de conferir maior segurança aos cirurgiões-dentistas na confecção e manutenção das próteses sobre implantes inclinados.

\section{METODOLOG I A}

A metodologia adotada foi a pesquisa bibliográfica, buscando-se a literatura já publicada a respeito do tema. Foram priorizados artigos e publicações em revistas, preferencialmente datados a partir do ano 2000, apesar de que os textos considerados clássicos nesse tema não foram desprezados.

Depois de feita a identificação das obras e autores, o levantamento bibliográfico, a localização e compilação foram feitos por meio da internet, com buscas nas principais bases de dados da área médica e odontológica. Para isso foi utilizado o cadastro disponibilizado pela universidade com a base de dados da CAPES, que possibilita a coleta de publicações restritas de bases como a PubMed, Scielo e BBO, além de disponibilizar na sua página de periódicos uma vasta gama de artigos.

\section{REVISÃO DA LITERATURA}

\section{Interface osso-implante}

Condições de carregamento desfavoráveis, como contato prematuro ou trauma oclusal, podem levar a falhas no tratamento reabilitador, pois favorecem o aparecimento de alterações mecânicas como tensões e deformações na interface osso-implante, que influenciam na resposta do tecido ósseo ${ }^{7}$.

As forças que atuam sobre os implantes possuem magnitude e direção; uma força de magnitude similar pode ter efeitos diferentes sobre a interface entre o implante e o tecido ósseo, dependendo da direção de aplicação da carga. Toda força pode
LITTUMA GJS

LOPEZ CAV

ENGLER MLPD

MAGINI RS

Meurer e

ANÁLISE

TRIDIMENSIONAL

DE ELEMENTOS

FINITOS DA

DISTRIBUIÇÃO

DE TENSÕES

EM IMPLANTES

INCLINADOS:

UMA REVISÃO DE

LITERATURA

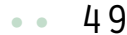

REV, ODONTOL.

UnIV. CID. São

PAULO

$2016 ; 29(1): 48-$

56 , JAN-ABR 
ITTUMA GJS

LOPEZ CAV

ENGLER MLPD

MAGINI RS

MEURER E

ANÁLISE

TRI DIMENS IONAL

DE ELEMENTOS

FINITOS DA

DISTRIBUIÇÃO

DE TENSÕES

EM IMPLANTES

INCLINADOS:

UMA REVISÃO DE LITERATURA

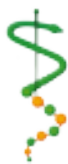

REV, ODONTOL.

UNIV, CID, SÃO

PAULO

$2016 ; 29(1): 48-$

56 , JAN-ABR
I SSN 1983-5183

dividir-se em uma combinação de componentes - sejam forças de compressão e tração, que atuam perpendicularmente à superfície ou plano de interesse; sejam de cisalhamento, que atuam paralelas à superfície. As forças compressivas tendem a manter a integridade da interface osso-implante, enquanto as forças de tração tendem a alterar ou abrir essa interface; da mesma maneira que as forças de cisalhamento. O osso é especialmente resistente às forças de compressão, porém resiste menos às forças de tração e notavelmente menos às de cisalhamento ${ }^{1}$.

As cargas oclusais também são classificadas como axiais e não axiais, sendo que as primeiras atuam perpendicularmente ao plano oclusal e são mais favoráveis (distribuem a tensão de forma mais equilibrada ao longo do implante), enquanto as cargas não axiais atuam em direção não perpendicular à superfície oclusal e exercem maiores gradientes de tensão no implante bem como no osso ${ }^{8}$.

A maneira como uma força é distribuída sobre uma superfície é chamada de tensão mecânica. Uma vez que um sistema comercial de implantes é selecionado, a única forma que o operador tem de controlar a tensão é conhecer a biomecânica que o desenho da prótese final, tamanho, número, angulação, diâmetro e comprimento dos implantes exercerão no conjunto ${ }^{1}$.

É necessário estabelecer uma relação entre as tensões internas que são geradas num implante e os tecidos circundantes sob o efeito de uma carga, assim como a posterior deformação que todo o sistema pode vir a sofrer. Ao se aplicar uma força sobre um corpo elástico é possível elaborar uma curva de carga perante a deformação; essa mesma curva pode prever a deformação que um determinado material sofrerá com a aplicação de uma carga, o qual se denomina Módulo de Elasticidade (E). A fórmula " $\mathrm{E}=(\sigma) /(\varepsilon)^{\prime}$ " permite concluir que quanto mais baixa for a deformação para uma determinada tensão maior será o módulo de elasticidade. A porcentagem de contato osso-implante é significantemente maior no osso cortical que no osso trabecular devido ao fato de que o osso cortical tem um maior módulo de elasticidade e, por consequência, maior resistência à deformação. Essa é a razão pela qual o osso cortical suporta mais cargas que o osso trabecular em situações clínicas ${ }^{1}$.

Segundo Bozkaya et al. ${ }^{9}$ (2004), a faIha óssea pode ser definida como a fratura local do osso causada por cargas locais excessivas. Portanto, para entender o que seria uma força excessiva capaz de comprometer o sucesso de um tratamento reabilitador, os autores Akça e Iplikçioglu $^{10}$ (2001) afirmaram que os processos biológicos como reabsorção ou remodelamento ósseo não podem ser previstos; no entanto, falhas ósseas acontecem quando os valores de tensão superam os limites de resistência do osso cortical em compressão (167Mpa) e em tração (121Mpa).

O osso é um material de alta complexidade, tendo propriedades anisotrópicas, heterogêneas e visco-elásticas ${ }^{11}$. Qualquer corpo sob a ação de forças externas (tração e compressão) apresenta uma deformação longitudinal $(\varepsilon)$, no sentido da aplicação dessas forças e também uma deformação no sentido transversal $(\varepsilon q)$. A relação entre os valores absolutos da deformação longitudinal e da transversal representa o coeficiente de Poisson, expresso na fórmula como " $V=\varepsilon / \varepsilon q^{\prime 12}$.

As propriedades mecânicas dos materiais possuem grande influência nos esforços e na distribuição de forças em uma estrutura. Essas propriedades podem ser caracterizadas como isotrópicas ou anisotrópicas: em um material isotrópico o comportamento mecânico é o mesmo em todas as direções, enquanto que um material anisotrópico apresentará diferentes propriedades quando medido em direções diferentes ${ }^{13}$.

\section{Análise dos Elementos Finitos}

O Método de Análise dos Elementos Finitos (MEF) é uma técnica numérica muito utilizada em engenharia para resolver problemas de mecânica contínua. $\mathrm{Na}$ implantodontia, durante mais de uma década, muitos pesquisadores têm aplicado o MEF para avaliar como se distribuem as forças em um implante dental e nos tecidos circundantes, e a sua simulação tem sido considerada comumente na literatura como material isotrópico homogêneo e linearmente elástico ${ }^{1}$. 
O MEF pode ser definido basicamente como uma técnica para obter a solução de um sistema mecânico através da divisão de um elemento de forma geométrica predeterminada em uma coleção de pequenos elementos, nos quais, os vários campos criados podem ser inseridos com características próprias ${ }^{14}$.

Os estudos de elementos finitos têm suas limitações já que as propriedades mecânicas e o comportamento não linear dos tecidos biológicos não podem ser previstos de forma precisa ${ }^{15}$.

Alguns fatores podem fazer com que as simulações no MEF se tornem irreais, tais como: o uso de cargas estáticas direcionadas axialmente; considerar as propriedades ósseas como homogêneas, lineares e isotrópicas, sabendo-se que são heterogêneas anisotrópicas e não lineares; e considerar a união implante/osso como perfeitamente estável quando ela é dinâmica $^{10,16}$.

Os resultados de MEF devem ser analisados com cautela, visto que características biológicas individuais como qualidade óssea, forças mastigatórias, características oclusais, presença de hábitos parafuncionais etc, são variáveis que limitam um completo estudo para comparar e implementar na prática clínica ${ }^{13}$.

\section{Implantes Inclinados}

A utilização de implantes inclinados surgiu como uma adaptação da técnica convencional em função de condições anatômicas e funcionais presentes na mandíbula e maxila ${ }^{17}$. De acordo com Moraes et al. ${ }^{18}$ (2002), variações na angulação dos implantes são condições indesejáveis, podendo ocasionar a potencialização das forças mastigatórias sobre o sistema.

Já foi relatado que a direção em que o implante é instalado está diretamente relacionada com a distribuição de tensões das cargas oclusais ${ }^{19} \mathrm{e}$, à medida que aumenta a angulação entre o corpo do implante e a carga, aumentam as tensões sobre todo o sistema prótese-implante-osso ${ }^{20}$.

Quando se aplica uma força na direção do longo eixo do implante, a tensão se concentra na crista óssea e a intensidade da tensão aumenta como resultado do posicionamento do implante: com uma angulação de $15^{\circ}$, a tensão na crista au- menta 25,9\%; em uma instalação do implante com angulação de $30^{\circ}$, a conexão pilar-implante e a interface osso-implante estão submetidas a uma tensão 50\% maior na inclinação do eixo longitudinal do implante $^{20}$.

O uso de implantes inclinados na crista residual pode gerar algumas vantagens clínicas, tais como: 1) torna possível instalar implantes mais longos, aumentando a área de contato osso-implante como a estabilidade primária; 2) cria-se uma ampliação da distância entre os implantes anteriores e posteriores o que resulta em uma melhor distribuição de forças; 3) a técnica reduz ou até elimina a necessidade de cantiléveres na prótese; 4) possibilita eliminar a necessidade de procedimentos de enxertia óssea, como levantamento do seio ou elevação da crista em regiões atróficas ${ }^{21}$.

\section{Estudos de Elementos Finitos com} Implantes Inclinados

Canay et al. ${ }^{22}$ (1996) analisaram a distribuição de tensões ao redor de implantes verticais e angulados instalados na região do primeiro molar mandibular sobre cargas verticais e horizontais. Os autores concluíram que, devido ao fato de que em um carregamento vertical as cargas transmitidas aos implantes verticais seguem o longo eixo do mesmo, valores de tensão de tração e compressão são quase simétricos. Já nos implantes angulados, valores de tensão compressiva são cinco vezes maiores em torno da região cervical e vestibular do implante, o que sugere que o uso de implantes angulados em regiões posteriores onde a tensão vertical é especialmente formada deve ser limitado.

Akça e Iplikçioglu ${ }^{10}$ (2001) avaliaram a influência de implantes alinhados de forma desordenada e a utilização de implantes de diâmetro largo alinhados de forma linear em uma mandíbula posterior edêntula. Os resultados demonstraram que os menores valores de tensão de compressão e tração foram encontrados nos implantes de maior diâmetro e posicionados de forma linear; quanto aos implantes alinhados de forma desordenada para vestibular e lingual, encontraram-se valores similares de tensão. Os autores concluíram que a instalação de implantes alinhados com
LITTUMA GJS

LOPEZ CAV

ENGLER MLPD

MAGINI RS

Meurer e

ANÁLISE

TRIDIMENSIONAL

DE ELEMENTOS

FINITOS DA

DI STRI BUIÇÃO

DE TENSÕES

EM IMPLANTES

INCLINADOS:

UMA REVISÃO DE

LITERATURA

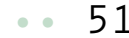

REV, ODONTOL.

UNIV, CID, SÃo

PAULO

$2016 ; 29(1): 48$

56 , JAN-ABR 
ITTUMA GJS LOPEZ CAV

ENGLER MLPD MAGINI RS MEURER E

ANÁLISE TRI D IMENS I ONAL DE ELEMENTOS FINITOS DA DISTRI BUIÇÃO DE TENSÕES EM IMPLANTES INCLINADOS: UMA REVISÃO DE LITERATURA

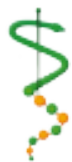

REV, ODONTOL.

UNIV. CID. SÃO

PAULO

$2016 ; 29(1): 48-$

56 , JAN-ABR
I SSN 1983-5183

maior diâmetro permite uma melhor distribuição de forças devido ao aumento da área de superfície e à massa que se contrapõe às forças transversais que causam momentos de flexão.

Las Casas et al. ${ }^{23}$ (2002) analisaram a distribuição de tensão na interface osso-implante ao longo da superfície mésio-distal e vestíbulo-lingual de dois tipos de implantes, um vertical e outro angulado. Os resultados mostraram uma maior concentração de tensão de tração e compressão na região cérvico-vestibular dos implantes angulados perante cargas verticais e um comportamento biomecânico similar em ambos os grupos diante de cargas horizontais. Os autores mencionam que, durante a função normal de mastigação, os componentes verticais de força são significantemente maiores que os componentes horizontais, porém, os resultados obtidos não limitam o desempenho clínico dos implantes inclinados.

Watanabe et al. ${ }^{24}$ (2003) avaliaram a influência da inclinação do implante, posição e direção de carga na distribuição de tensão. Os autores concluíram que pelo fato de a tensão ao redor do osso ser bastante influenciada pela posição, direção de carga e inclinação do implante, deve-se ter muito cuidado para que a carga seja aplicada de forma vertical ou em último caso próxima ao longo eixo do implante. No entanto, é impossível definir o limite de inclinação que os implantes podem ter sem causar dano considerável ao osso.

Satoh et al. ${ }^{25}$ (2006) verificaram a distribuição de tensões ao redor de dois implantes instalados na região da mandíbula sob diferentes inclinações, tendo como conceito que, em um arco dental, os dentes posteriores tendem a inclinar-se numa direção mesial e lingual em relação à inclinação natural da curva de Spee. Os resultados mostraram valores de tensão maiores no osso de suporte e na área cérvico-mesial e distal dos implantes sem inclinação axial em relação aos implantes distais inclinados $\left(10^{\circ}\right)$ em sentido mesial e mésio-lingual, sugerindo, ao final, que uma inclinação mesial e mésio-lingual do implante distal pode ser considerada uma vantagem biomecânica sempre que as cargas sejam perpendiculares à superfície oclusal.
Çaglar et al. ${ }^{25}$ (2006) avaliaram o efeito da inclinação mésio-distal dos implantes na distribuição de forças em uma prótese fixa implantossuportada instalada na região posterior edêntula da maxila. Os resultados mostraram que os menores valores de tensão foram observados nas cargas verticais, e os maiores valores, com o carregamento oblíquo. As tensões ficaram concentradas na área de osso cortical nos três tipos de carga. Os valores encontrados na região cervical do implante foram maiores nas áreas vestibular e palatina, em relação às áreas mesial e distal. Devido à inclinação do implante, as tensões na região distal foram maiores que nas regiões mesial, vestibular e palatina do pescoço do implante na área do molar. Para as cargas verticais, a tensão no pescoço do implante na região de pré-molares aumentou 3,5 vezes mais com a segunda configuração e duas vezes mais com os carregamentos oblíquos e horizontais. No modelo com cantiléver, a tensão foi maior no implante adjacente ao cantiléver. O maior valor de tensão encontrado no estudo foi de 194,1 Mpa após o carregamento oblíquo.

Cruz et al. ${ }^{26}$ (2009) analisaram a distribuição de forças ao redor de dois sistemas de próteses implantossuportadas, ambos com implantes alinhados na crista alveolar, em uma configuração reta dentro do osso ou inclinada. Os resultados mostraram que a distribuição de tensão foi similar nos dois sistemas, apresentando uma maior concentração de tensão perante uma carga vertical na área mesial e distal do pescoço do primeiro implante reto no sistema alinhado e no terceiro implante angulado do segundo sistema. Além disso, nenhuma concentração de tensão considerável foi encontrada no sistema de implantes angulados ao longo do corpo, ângulos internos, externos ou ápice, diante de cargas verticais e horizontais. Os autores concluíram que o deslocamento intraósseo criado com implantes angulados não altera a distribuição de tensão e pode ser uma opção para aumentar a estabilidade biomecânica, reduzindo o grau de tensões sobre cargas oblíquas.

Silva et al. ${ }^{6}$ (2010) avaliaram, em um modelo de maxila, em condições homogêneas, isotrópicas e elásticas, o compor- 
tamento biomecânico de dois tipos de próteses implantossuportadas - a primeira um sistema tipo "All-on-4" e a segunda, uma prótese fixa de 6 implantes, ambas com os últimos implantes inclinados. Os autores concluíram que, em todas as situações de carga, o pico de tensão localizou-se na região cervical do implante inclinado distalmente, apresentando menores valores no sistema de 6 implantes, pelo que os autores sugerem que, em situações de risco como bruxismo e baixa qualidade óssea, por exemplo, um número maior de implantes poderia ser necessário para dar suporte protético adequado. Por outro lado, sugere-se minimizar ou evitar o uso de cantiléveres, pois sua presença aumenta a tensão no implante distal independentemente do número de implantes.

Bevilacqua et al. ${ }^{27}$ (2011) avaliaram a transmissão de carga no osso peri-implantar maxilar usando diferentes inclinações de implantes e larguras do cantiléver, mediante a instalação na maxila edêntula. Os resultados mostraram que os maiores valores de tensão foram registrados no osso cortical com os implantes verticais apresentando 75Mpa no implante distal e $35 \mathrm{Mpa}$ no implante mesial. Consequentemente, nos implantes distais angulados e com uma redução do cantiléver posterior, os valores de tensão diminuíram até 65.3Mpa no implante distal, e 28.6 Mpa no implante mesial com a configuração de $15^{\circ}$; 39.4 Mpa e 16.6 Mpa na configuração com $30^{\circ}$ e 19.9 Mpa e $7.8 \mathrm{Mpa}$ com a configuração de $45^{\circ}$. Da mesma forma, na estrutura metálica da prótese fixa, o maior valor de tensão transmitido foi de 95.0 Mpa com os implantes verticais e de 13.7 Mpa com os implantes inclinados a $45^{\circ}$. Os autores concluíram que o uso de implantes inclinados reduz as tensões no osso peri-implantar e na estrutura metálica como resultado da redução do tamanho do cantiléver e do aumento do comprimento dos implantes.

\section{I SCUSSÃO}

De acordo com Brunski ${ }^{7}$ (1988), fatores mecânicos como as micromovimentações, tensões e deformações na interface osso-implante podem influenciar na resposta do tecido ósseo aos implantes.
Considerando-se que a única forma que o operador tem de controlar o esforço experimentado pelos tecidos é através do controle das tensões, e que um maior ângulo entre o corpo do implante e a carga aumenta a magnitude de tensão de acordo com Misch ${ }^{1}$ (2006); concorda-se com Almeida et al. ${ }^{3}$ (2008) em que o fator chave de sucesso ou falha na utilização de implantes é como as tensões são transferidas ao osso peri-implantar.

Neste trabalho, comparando os resultados das diferentes AEF em relação à influência da inclinação dos implantes sob a tensão no osso, pode-se observar que a maioria dos autores considerou que a tensão aumenta proporcionalmente à angulação dos implantes ${ }^{22,1,10,23,24,25,6}$.

Em concordância, Watanabe et al. ${ }^{24}$ (2003) e Satoh et al. ${ }^{19}$ (2005) perceberam que os valores de tensão foram superiores mediante a inclinação dos implantes e afirmaram que deve-se ter muito cuidado para que a carga seja aplicada de forma vertical ou, em último caso, próxima ao longo eixo do implante.

Os resultados de Canay et al..22 (1996) concordam com os obtidos por Las Casas et al. ${ }^{23}$ (2002), que mostraram uma maior concentração de forças de tensão e compressão na região cérvico-vestibular dos implantes angulados para cargas verticais e um comportamento biomecânico similar em ambos os grupos para cargas horizontais; no entanto, é importante mencionar que ainda que os valores máximos de tensão frente a cargas horizontais nos dois trabalhos sejam similares para ambos os grupos, esses valores foram elevados e superiores na utilização de implantes angulados, superando, no estudo feito por Canay et al.22 (1996), o limite máximo de resistência de tensão do osso cortical (121Mpa) descrito por Akça e Iplikçioglu ${ }^{10}$ (2001), aumentando consideravelmente o risco de possíveis falhas ósseas.

Esses resultados diferem dos obtidos por Cruz et al. ${ }^{26}$ (2009), que afirmaram que o uso de implantes angulados não produz uma maior concentração de tensão nos ângulos internos ou externos do implante perante cargas verticais e horizontais, concordando com Satoh et al. ${ }^{19}$ (2005), que relataram que um desloca-
LITTUMA GJS

LOPEZ CAV

ENGLER MLPD

MAGINI RS

MEURER E

ANÁLISE

TRIDIMENSIONAL

DE ELEMENTOS

FINITOS DA

DISTRI BUIÇÃO

DE TENSÕES

EM IMPLANTES

INCLINADOS:

UMA REVISÃo DE

LITERATURA

REV, ODONTOL

UnIV. CID. São

PAULO

2016; 29(1): 48 -

56, JAN-ABR 
LITTUMA GJS LOPEZ CAV

ENGLER MLPD

MAGINI RS

MEURER E

ANÁLISE

TRI DIMENS IONAL

DE ELEMENTOS

FINITOS DA

DISTR I BU I ÇÃO

DE TENSÕES

EM IMPLANTES

INCLINADOS:

UMA REVISÃO DE

LITERATURA

54

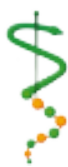

REV, ODONTOL.

UNIV. CID, SÃO

PAULO

$2016 ; 29(1): 48-$

$56, J A N-A B R$ mento intencional dos implantes pode ser considerado uma opção para aumentar a estabilidade biomecânica.

Akça e Iplikçioglu ${ }^{10}$ (2001) concluíram, no entanto, que menores valores de tensão compressiva e de tração podem ser observados no osso cortical, na região cervical dos implantes alinhados com maior diâmetro em relação aos que apresentavam algum deslocamento; isso ocorre devido ao aumento da área de superfície e da massa, que se contrapõem às forças transversais que causam momentos de flexão.

Com base nos resultados do trabalho de Çaglar et al. ${ }^{25}$ (2006) perante diferentes tipos de carregamento (vertical, oblíquo ou horizontal), os maiores valores de tensão foram encontrados com cargas oblíquas, concordando com Vidyasagar e $\operatorname{Apse}^{8}$ (2003), que relataram que cargas não axiais que atuam em direção não perpendicular à superfície oclusal exercem gradientes de tensão no implante assim como no osso; fator que pode ser ainda mais prejudicial no caso de uma prótese fixa com dois implantes e uma extensão em cantiléver onde, segundo os autores, valores de tensão aumentaram duas vezes na área adjacente à região do cantiléver.

Silva et al. ${ }^{6}$ (2010) concordam com Bevilacqua et al. ${ }^{27}$ (2011), em minimizar ou evitar o uso de cantiléveres na prótese fixa implantossuportada porque sua presença aumenta a tensão nos implantes angulados; todavia, Silva et al. ${ }^{6}$ (2010) sugerem que um número maior de implantes poderia ser necessário para se ter suporte adequado em situações de risco. Bevilacqua et al. ${ }^{27}$ (2011) consideram que, utilizando-se implantes de maior diâmetro e comprimento, é possível diminuir a tensão no osso peri-implantar e infraestrutura metálica de suporte.

De acordo com os resultados dos estudos apresentados por Canay et al. ${ }^{22}$ (1996) e Çaglar et al. ${ }^{25}$ (2006), a maior concentração de estresse encontra-se no osso cortical, fator que, segundo Misch ${ }^{1}$, é resultado da maior porcentagem de contato ossoimplante nessa área, além do maior mó- dulo de elasticidade que permite maior resistências às cargas.

Um fator a ser considerado é que o método de AEF também tem suas limitações. Nos estudos de análise, é considerada uma completa osseointegração do implante e nas simulações as propriedades do osso são consideradas como isotrópicas, homogêneas e lineares, fato que é irreal, pois De Tolla et al. ${ }^{16}$ (2000) mencionam a união osso-implante como dinâmica, além de o osso apresentar propriedades anisotrópicas, heterogêneas e não lineares.

\section{CONCLUSÃO}

Analisando-se os parâmetros e resultados de análise de elementos finitos no presente trabalho, nota-se que a utilização de implantes inclinados aumenta consideravelmente a concentração de tensões no osso cortical peri-implantar. No entanto, o deslocamento intencional pode ser considerado como uma opção de tratamento para aumentar a estabilidade biomecânica das próteses, sempre que as cargas sejam transmitidas perpendicularmente à superfície oclusal. Não foi possível determinar o grau de tensão que pode desencadear reabsorção óssea e remodelação quando da utilização de implantes inclinados; porém, perante a aplicação de forças horizontais sobre os implantes angulados, elevados valores de tensão poderiam comprometer os limites de resistência do osso cortical, podendo desencadear falhas ósseas.

A análise de elementos finitos tem sido utilizada como forma de prever com maior precisão o efeito da tensão aplicada sobre os implantes e ao redor do tecido ósseo. Contudo, é difícil predizer através desse método o comportamento real que essa estrutura viva pode ter, pelas propriedades anisotrópicas, heterogêneas e não lineares que apresenta; portanto, recomenda-se a necessidade de se criarem modelos mais específicos e avançados que permitam ser extrapolados para as situações clínicas. 
1. Misch CE. Prótese sobre implantes. São Paulo: Santos; 2006.

2. Eskitascioglu G, Usumez A, Sevimay M, Soykan E, Unsal E. The influence of occlusal loading location on stresses transferred to implant-supported prostheses and supporting bone: A three-dimensional finite element study. The Journal of prosthetic dentistry 2004 Feb;91(2):144-50.

3. Almeida EO, Pellizzer EP. Biomecânica em prótese sobre implante relacionada às inclinações das cúspides e às angulações dos implantes osseointegrados: revisão de literatura. $\operatorname{Rev}$ odontol UNESP (Online) 2008 out-dez;37(4): 321-7.

4. Geng JP, Tan KB, Liu GR. Application of finite element analysis in implant dentistry: a review of the literature. The Journal of prosthetic dentistry 2001 Jun;85(6):585-98.

5. Rubo JH, Capello Souza EA. Finite-element analysis of stress on dental implant prosthesis. Clinical implant dentistry and related research 2010 Jun 01;12(2):105-13.

6. Silva GC, Mendonca JA, Lopes LR, Landre J, Jr. Stress patterns on implants in prostheses supported by four or six implants: a three-dimensional finite element analysis. The International journal of oral \& maxillofacial implants 2010 Mar-Apr;25(2):239-46.

7. Brunski JB. Biomaterials and biomechanics in dental implant design. The International journal of oral \& maxillofacial implants 1988 Summer;3(2):85-97.

8. Vidyasagar L, Apse P. Biological response to dental implant loading/overloading. Implant overloading: empiricism or science? Stomatol, Baltic Dent Maxillofac J 2003 5(3):83-9.
9. Bozkaya D, Muftu S, Muftu A. Evaluation of load transfer characteristics of five different implants in compact bone at different load levels by finite elements analysis. The Journal of prosthetic dentistry 2004 Dec;92(6):52330.

10. Akça K, Iplikçioglu H. Finite element stress analysis of the influence of staggered versus straight placement of dental implants. The International journal of oral \& maxillofacial implants 2001 Sep-Oct; 16(5):722-30.

11. Erdmann B, Kober C, Lang J, Deuflhard P, Zeilhofer H-F, Sader R. Efficient and reliable finite element methods for simulation of the human mandible. Konrad-Zuse-Zentrum für Informationstechnik Berlin 2001 JUL.;7(1):114.

12. Silva MG. Influência da esplintagem de restaurações protéticas fixas e do número de implantes na distribuição de tensões em mandíbula edentada posterior: análise de elementos finitos [Dissertação]. São Paulo: Faculdade de Odontologia da USP; 2005.

13. Blatt M, Butignon LE, Bonachela WC. Análise de elementos finitos aplicados a implantodontia: uma nova realidade do virtual para o real. Innov Implant J 2006 dez;1(2):53-62.

14. Rubo JH, Souza EAC. Métodos computacionais aplicados à bioengenharia: soluçäo de problemas de carregamento em próteses sobre implantes. Rev Fac Odontol Bauru 2001 jul-dez ;9((3/4)):97-103.

15. Geramy A, Morgano SM. Finite element analysis of three designs of an implant-supported molar crown. The Journal of prosthetic dentistry 2004 Nov;92(5):434-40.

16. De Tolla DH, Andreana S, Patra A, Buhite R, Comella B. The role of the finite element model in dental implants. J Oral Imp/ 2000 26(2):77-81.
LITTUMA GJS

LOPEZ CAV

ENGLER MLPD

MAGINI RS

Meurer e

ANÁLISE

TRIDIMENSIONAL

DE ELEMENTOS

FINITOS DA

DI STRI BUIÇÃO

DE TENSÕES

EM IMPLANTES

INCLINADOS:

UMA REVISÃo DE

LITERATURA

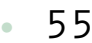

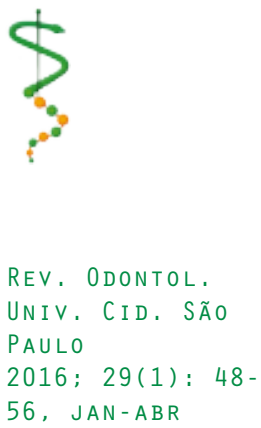


LITTUMA GJS LOPEZ CAV

ENGLER MLPD

MAGINI RS

MEURER E

ANÁLISE

TRIDIMENSIONAL

DE ELEMENTOS

FINITOS DA

DISTRIBUIÇÃO

DE TENSÕES

EM IMPLANTES

INCLINADOS:

UMA REVISÃO DE

LITERATURA
17. Krekmanov L, Kahn M, Rangert B, Lindstrom $\mathrm{H}$. Tilting of posterior mandibular and maxillary implants for improved prosthesis support. The International journal of oral \& maxillofacial implants 2000 May-Jun;15(3):405-14.

18. Moraes MCCSB, Moraes EJ, Elias CN. Análise de tensões em implantes osseointegrados por elementos finitos: variação da inclinação da cúspide e largura da mesa oclusal. Rev bras Implant 2002 abr-jun ;8(2):21-6.

19. Satoh T, Maeda Y, Komiyama Y. Biomechanical rationale for intentionally inclined implants in the posterior mandible using 3D finite element analysis. The International journal of oral \& maxillofacial implants 2005 Jul-Aug;20(4):533-9.

20. Misch CE. Implantes dentários contemporâneos. São Paulo: Santos; 2000.

21. Testori T, Del Fabbro M, Capelli M, Zuffetti F, Francetti L, Weinstein RL. Immediate occlusal loading and tilted implants for the rehabilitation of the atrophic edentulous maxilla: 1-year interim results of a multicenter prospective study. Clinical oral implants research 2008 Mar;19(3):227-32.

22. Canay S, Hersek N, Akpinar I, Asik Z. Comparison of stress distribution around vertical and angled implants with finite-element analysis. Quintessence international (Berlin, Germany: 1985) 1996 Sep;27(9):591-8.
23. Las Casas EB, Ferreira PC, Cimini Jr CA, Toledo EM, Barra LPS, Cruz M. A comparison between the stress distribution in angled and vertical implants. MECOM 2002 Oct.;21(10):2475-86.

24. Watanabe F, Hata Y, Komatsu S, Ramos TC, Fukuda H. Finite element analysis of the influence of implant inclination, loading position, and load direction on stress distribution. Odontology 2003 Sep;91(1):31-6.

25. Çaglar A, Aydin C, Ozen J, Yilmaz C, Korkmaz T. Effects of mesiodistal inclination of implants on stress distribution in implant-supported fixed prostheses. The International journal of oral \& maxillofacial implants 2006 Jan-Feb;21(1):36-44.

26. Cruz M, Wassall T, Toledo EM, Da Silva Barra LP, Cruz S. Finite element stress analysis of dental prostheses supported by straight and angled implants. The International journal of oral \& maxillofacial implants 2009 May-Jun;24(3):391-403.

27. Bevilacqua $M$, Tealdo T, Menini $M$, Pera F, Mossolov A, Drago C, et al. The influence of cantilever length and implant inclination on stress distribution in maxillary implant-supported fixed dentures. The Journal of prosthetic dentistry 2011 Jan;105(1):5-13.

Recebido em 20/09/2016

Aceito em 01/12/2016

\footnotetext{
REV. ODONTOL.

UNIV, CID. SÃO PAULO
} $2016 ; 29(1): 48-$ 56 , JAN-ABR 